\title{
FIXED POINT RESULTS SATISFYING RATIONAL TYPE CONTRACTIVE CONDITIONS IN COMPLEX VALUED METRIC SPACES - REVISITED
}

\author{
Mian Bahadur Zada, Muhammad Sarwar*
}

\begin{abstract}
In our previous work titled "Fixed Point Results Satisfying Rational type Contractive Conditions in Complex Valued Metric Spaces"[Ann. Math. Sil. 30 (2016), 89-110], some errors has been made in the main results (Theorem 3.1, Theorem 3.7 and Theorem 3.22), that may misguide the readers. This note provides corrections of these errors.
\end{abstract}

Examining the paper [1], we have found some minor errors in Theorems 3.1, 3.7, 3.22 and Lemma 3.21, which we eliminate here.

In Theorem 3.1 we slightly modify the last component of the assumption (ii). Moreover, the denominators of the rational expressions should be corrected as:

$1+d(z, w)+d(w, L z)$ instead of $1+d(z, w) d(w, L z)$ in line 9 on page 94 ,

$1+d\left(z_{n}, z_{n+1}\right)+d\left(z_{n+1}, L z_{n}\right)$ instead of $1+d\left(z_{n}, z_{n+1}\right) d\left(z_{n+1}, L z_{n}\right)$ in line 2 on page 95 ,

$1+d\left(z_{n}, z_{n+1}\right)+d\left(z_{n+1}, z_{n+1}\right)$ instead of $1+d\left(z_{n}, z_{n+1}\right) d\left(z_{n+1}, z_{n+1}\right)$ in line 9 on page 95 ,

$1+d\left(z_{n}, t\right)+d\left(t, L z_{n}\right)$ instead of $1+d\left(z_{n}, t\right) d\left(t, L z_{n}\right)$ in line 3 on page 97,

Received: 24.06.2016. Accepted: 18.11.2016. Published online: 07.02.201\%.

(2010) Mathematics Subject Classification: 47H10, 54H25.

Key words and phrases: complex valued metric spaces, common fixed points, self-maps, Cauchy sequence, contractive conditions.

*Corresponding author. 
$1+d\left(z_{n}, t\right)+d\left(t, z_{n+1}\right)$ instead of $1+d\left(z_{n}, t\right) d\left(t, z_{n+1}\right)$ in line 9 on page 97 ,

$1+d\left(t, t^{*}\right)+d\left(t^{*}, L t\right)$ instead of $1+d\left(t, t^{*}\right) d\left(t^{*}, L t\right)$ in line 3 on page 98 ,

$1+2 d\left(t, t^{*}\right)$ instead of $1+d\left(t, t^{*}\right) d\left(t^{*}, t\right)$ in line 6 on page 98 ,

and the numerators of the rational expressions should be corrected as $d\left(t, L t^{*}\right)[1+d(t, L t)]$ instead of $d(t, L t)[1+d(t, L t)]$ in line 2 on page 98 , $d\left(t, L t^{*}\right)\left[1+d\left(t^{*}, L t\right)\right]$ instead of $d(t, L t)\left[1+d\left(t^{*}, L t\right)\right]$ in line 3 on page 98 , $d\left(t, t^{*}\right)\left[1+d\left(t^{*}, t\right)\right]$ instead of $d\left(t, t^{*}\right)$ in line 6 on page 98 .

The correct version of Theorem 3.1 ([1]) reads as follows.

TheOREm 1. Let $(X, d)$ be a complete complex valued metric space and $\lambda_{i}: X \times X \rightarrow[0,1), i=1,2, \ldots, 8$. Assume that $L: X \rightarrow X$ is a self-map such that for all $z, w \in X$ the following conditions are satisfied:

(i) $\lambda_{i}(L z, w) \leq \lambda_{i}(z, w)$ and $\lambda_{i}(z, L w) \leq \lambda_{i}(z, w), i=1, \ldots, 8$;

(ii) $d(L z, L w) \precsim \lambda_{1}(z, w) d(z, w)+\lambda_{2}(z, w) d(z, L w)$

$$
\begin{aligned}
& +\lambda_{3}(z, w) d(w, L z)+\lambda_{4}(z, w) d(z, L z) \\
& +\lambda_{5}(z, w) d(w, L w)+\lambda_{6}(z, w) \frac{d(w, L w)[1+d(z, L z)]}{1+d(z, w)} \\
& +\lambda_{7}(z, w) \frac{d(z, L w)[1+d(z, L z)]}{1+d(z, w)} \\
& +\lambda_{8}(z, w) \frac{d(z, L w)[1+d(w, L z)]}{1+d(z, w)+d(w, L z)}
\end{aligned}
$$

where

$$
\lambda_{1}(z, w)+\lambda_{3}(z, w)+\sum_{i=4}^{6} \lambda_{i}(z, w)+2\left[\lambda_{2}(z, w)+\lambda_{7}(z, w)+\lambda_{8}(z, w)\right]<1 .
$$

Then the mapping $L$ has a unique fixed point in $X$.

Proof. Let $z_{0} \in X$ and let us construct a sequence $\left\{z_{n}\right\}$ by the rule

$$
L z_{n}=z_{n+1}, \quad n=0,1,2, \ldots
$$

First we show that $\left\{z_{n}\right\}$ is a Cauchy sequence in $X$. For this, consider

$$
d\left(z_{n+1}, z_{n+2}\right)=d\left(L z_{n}, L z_{n+1}\right) .
$$


By using condition (ii) of Theorem 1 with $z=z_{n}$ and $w=z_{n+1}$, we have

$$
\begin{aligned}
d\left(z_{n+1}, z_{n+2}\right) \precsim & \lambda_{1}\left(z_{n}, z_{n+1}\right) d\left(z_{n}, z_{n+1}\right)+\lambda_{2}\left(z_{n}, z_{n+1}\right) d\left(z_{n}, L z_{n+1}\right) \\
& +\lambda_{3}\left(z_{n}, z_{n+1}\right) d\left(z_{n+1}, L z_{n}\right)+\lambda_{4}\left(z_{n}, z_{n+1}\right) d\left(z_{n}, L z_{n}\right) \\
& +\lambda_{5}\left(z_{n}, z_{n+1}\right) d\left(z_{n+1}, L z_{n+1}\right) \\
& +\lambda_{6}\left(z_{n}, z_{n+1}\right) \frac{d\left(z_{n+1}, L z_{n+1}\right)\left[1+d\left(z_{n}, L z_{n}\right)\right]}{1+d\left(z_{n}, z_{n+1}\right)} \\
& +\lambda_{7}\left(z_{n}, z_{n+1}\right) \frac{d\left(z_{n}, L z_{n+1}\right)\left[1+d\left(z_{n}, L z_{n}\right)\right]}{1+d\left(z_{n}, z_{n+1}\right)} \\
& +\lambda_{8}\left(z_{n}, z_{n+1}\right) \frac{d\left(z_{n}, L z_{n+1}\right)\left[1+d\left(z_{n+1}, L z_{n}\right)\right]}{1+d\left(z_{n}, z_{n+1}\right)+d\left(z_{n+1}, L z_{n}\right)}
\end{aligned}
$$

Using now (1) and condition (i) of Theorem 1 , we get

$$
\begin{aligned}
\left|d\left(z_{n+1}, z_{n+2}\right)\right| \leq & \lambda_{1}\left(z_{0}, z_{1}\right)\left|d\left(z_{n}, z_{n+1}\right)\right|+\lambda_{2}\left(z_{0}, z_{1}\right)\left|d\left(z_{n}, z_{n+1}\right)\right| \\
& +\lambda_{2}\left(z_{0}, z_{1}\right)\left|d\left(z_{n+1}, z_{n+2}\right)\right|+\lambda_{4}\left(z_{0}, z_{1}\right)\left|d\left(z_{n}, z_{n+1}\right)\right| \\
& +\lambda_{5}\left(z_{0}, z_{1}\right)\left|d\left(z_{n+1}, z_{n+2}\right)\right|+\lambda_{6}\left(z_{0}, z_{1}\right)\left|d\left(z_{n+1}, z_{n+2}\right)\right| \\
& +\lambda_{7}\left(z_{0}, z_{1}\right)\left|d\left(z_{n}, z_{n+1}\right)\right|+\lambda_{7}\left(z_{0}, z_{1}\right)\left|d\left(z_{n+1}, z_{n+2}\right)\right| \\
& +\lambda_{8}\left(z_{0}, z_{1}\right)\left|d\left(z_{n}, z_{n+1}\right)\right|+\lambda_{8}\left(z_{0}, z_{1}\right)\left|d\left(z_{n+1}, z_{n+2}\right)\right| .
\end{aligned}
$$

Finally, we get

$$
\left|d\left(z_{n+1}, z_{n+2}\right)\right| \leq h\left|d\left(z_{n}, z_{n+1}\right)\right|
$$

where

$$
h=\frac{\lambda_{1}\left(z_{0}, z_{1}\right)+\lambda_{2}\left(z_{0}, z_{1}\right)+\lambda_{4}\left(z_{0}, z_{1}\right)+\lambda_{7}\left(z_{0}, z_{1}\right)+\lambda_{8}\left(z_{0}, z_{1}\right)}{1-\left(\lambda_{2}\left(z_{0}, z_{1}\right)+\lambda_{5}\left(z_{0}, z_{1}\right)+\lambda_{6}\left(z_{0}, z_{1}\right)+\lambda_{7}\left(z_{0}, z_{1}\right)+\lambda_{8}\left(z_{0}, z_{1}\right)\right)} .
$$

Similarly,

$$
\left|d\left(z_{n}, z_{n+1}\right)\right| \leq h\left|d\left(z_{n-1}, z_{n}\right)\right|
$$

Consequently,

$$
\left|d\left(z_{n+2}, z_{n+1}\right)\right| \leq h\left|d\left(z_{n+1}, z_{n}\right)\right| \leq h^{2}\left|d\left(z_{n}, z_{n-1}\right)\right| \leq \ldots \leq h^{n+1}\left|d\left(z_{1}, z_{0}\right)\right| .
$$


Now, for $m>n$,

$$
d\left(z_{n}, z_{m}\right) \precsim d\left(z_{n}, z_{n+1}\right)+d\left(z_{n+1}, z_{n+2}\right)+\ldots+d\left(z_{m-1}, z_{m}\right) .
$$

Thus $\left|d\left(z_{n}, z_{m}\right)\right| \leq \frac{h^{n}}{1-h}\left|d\left(z_{1}, z_{0}\right)\right|$, which implies that $\lim _{n \rightarrow \infty}\left|d\left(z_{n}, z_{m}\right)\right|=0$. Hence, $\left\{z_{n}\right\}$ is a Cauchy sequence. But $X$ is complete, so there exists $t \in X$ such that $z_{n} \rightarrow t$ as $n \rightarrow \infty$.

Next, we show that $t$ is a fixed point of $L$. For this, assume that $L t \neq t$. Then

$$
d(t, L t) \precsim d\left(t, L z_{n}\right)+d\left(L z_{n}, L t\right) .
$$

By applying conditions (i), (ii) of Theorem 1 and equation (1), equation (2) becomes

$$
\begin{aligned}
d(t, L t) \precsim & d\left(t, z_{n+1}\right)+\lambda_{1}\left(z_{0}, t\right) d\left(z_{n}, t\right)+\lambda_{2}\left(z_{0}, t\right) d\left(z_{n}, L t\right) \\
& +\lambda_{3}\left(z_{0}, t\right) d\left(t, z_{n+1}\right)+\lambda_{4}\left(z_{0}, t\right) d\left(z_{n}, z_{n+1}\right) \\
& +\lambda_{5}\left(z_{0}, t\right) d(t, L t)+\lambda_{6}\left(z_{0}, t\right) \frac{d(t, L t)\left[1+d\left(z_{n}, z_{n+1}\right)\right]}{1+d\left(z_{n}, t\right)} \\
& +\lambda_{7}\left(z_{0}, t\right) \frac{d\left(z_{n}, L t\right)\left[1+d\left(z_{n}, z_{n+1}\right)\right]}{1+d\left(z_{n}, t\right)} \\
& +\lambda_{8}\left(z_{0}, t\right) \frac{d\left(z_{n}, L t\right)\left[1+d\left(t, z_{n+1}\right)\right]}{1+d\left(z_{n}, t\right)+d\left(t, z_{n+1}\right)} .
\end{aligned}
$$

Taking limit as $n \rightarrow \infty$, we get

(3) $d(t, L t) \precsim\left[\lambda_{2}\left(z_{0}, t\right)+\lambda_{5}\left(z_{0}, t\right)+\lambda_{6}\left(z_{0}, t\right)+\lambda_{7}\left(z_{0}, t\right)+\lambda_{8}\left(z_{0}, t\right)\right] d(t, L t)$,

which is not possible. Thus $L t=t$. Hence $t$ is a fixed point of $L$.

For uniqueness, let $t^{*} \neq t$ be another fixed point of $L$. Then putting $z=t$ and $w=t^{*}$ in condition (ii) of Theorem 1 , we get

$$
\begin{aligned}
d\left(L t, L t^{*}\right) \precsim & \lambda_{1}\left(t, t^{*}\right) d\left(t, t^{*}\right)+\lambda_{2}\left(t, t^{*}\right) d\left(t, L t^{*}\right)+\lambda_{3}\left(t, t^{*}\right) d\left(t^{*}, L t\right) \\
& +\lambda_{4}\left(t, t^{*}\right) d(t, L t)+\lambda_{5}\left(t, t^{*}\right) d\left(t^{*}, L t^{*}\right) \\
& +\lambda_{6}\left(t, t^{*}\right) \frac{d\left(t^{*}, L t^{*}\right)[1+d(t, L t)]}{1+d\left(t, t^{*}\right)}+\lambda_{7}\left(t, t^{*}\right) \frac{d\left(t, L t^{*}\right)[1+d(t, L t)]}{1+d\left(t, t^{*}\right)} \\
& +\lambda_{8}\left(t, t^{*}\right) \frac{d\left(t, L t^{*}\right)\left[1+d\left(t^{*}, L t\right)\right]}{1+d\left(t, t^{*}\right)+d\left(t^{*}, L t\right)} .
\end{aligned}
$$


Thus

$$
\begin{aligned}
\left|d\left(t, t^{*}\right)\right| \leq & \lambda_{1}\left(t, t^{*}\right)\left|d\left(t, t^{*}\right)\right|+\lambda_{2}\left(t, t^{*}\right)\left|d\left(t, t^{*}\right)\right|+\lambda_{3}\left(t, t^{*}\right)\left|d\left(t^{*}, t\right)\right| \\
& +\lambda_{7}\left(t, t^{*}\right)\left|d\left(t, t^{*}\right)\right|+\lambda_{8}\left(t, t^{*}\right)\left|\frac{d\left(t, t^{*}\right)\left[1+d\left(t^{*}, t\right)\right]}{1+d\left(t, t^{*}\right)+d\left(t^{*}, t\right)}\right| \\
\leq & {\left[\lambda_{1}\left(t, t^{*}\right)+\lambda_{2}\left(t, t^{*}\right)+\lambda_{3}\left(t, t^{*}\right)+\lambda_{7}\left(t, t^{*}\right)+\lambda_{8}\left(t, t^{*}\right)\right]\left|d\left(t, t^{*}\right)\right|, }
\end{aligned}
$$

which is not possible. Hence $t$ is a unique fixed point of $L$.

In the proof of Theorem 3.7 the denominator of the rational expressions should be corrected as

$1+d\left(L z_{2 k-1}, z_{2 k-1}\right)+d\left(K L z_{2 k-1}, L z_{2 k-1}\right)$ instead of $1+d\left(L z_{2 k-1}, z_{2 k-1}\right)$ in lines $8-11,16,17$ on page 100 and lines 1,2 on page 101 ,

$1+d\left(z_{2 k-2}, K z_{2 k-2}\right)+d\left(K z_{2 k-2}, L K z_{2 k-2}\right)$ instead of $1+d\left(z_{2 k-2}, K z_{2 k-2}\right)$ in lines $8-11$ on page 102 ,

$1+d\left(z_{2 k-2}, z_{2 k-1}\right)+d\left(z_{2 k-1}, z_{2 k}\right)$ instead of $1+d\left(z_{2 k-2}, z_{2 k-1}\right)$ in line 17 on page 102 and lines 1, 2 on page 103,

$1+d\left(t, z_{2 n+1}\right)+d\left(K t, L z_{2 n+1}\right)$ instead of $1+d\left(t, z_{2 n+1}\right)$ in line 21 on page 104 and lines $1-3$ on page 105 ,

$1+d\left(t, z_{2 n+1}\right)+d\left(K t, z_{2 n+2}\right)$ instead of $1+d\left(t, z_{2 n+1}\right)$ in lines $10-13$ on page 105 ,

$1+d\left(t, t^{*}\right)+d\left(K t, L t^{*}\right)$ instead of $1+d\left(t, t^{*}\right)$ in lines $5-8$ on page 106 ,

$1+d\left(t, t^{*}\right)+d\left(t, t^{*}\right)$ instead of $1+d\left(t, t^{*}\right)$ in line 11 on page 106.

We rewrite lines $16-20$ on page 101 as

$$
\left|d\left(z_{2 k+1}, z_{2 k}\right)\right| \leq h_{0}\left|d\left(z_{2 k}, z_{2 k-1}\right)\right|
$$

where

$$
h_{0}=\frac{\lambda_{1}\left(z_{0}, z_{1}\right)+\lambda_{5}\left(z_{0}, z_{1}\right)+\lambda_{6}\left(z_{0}, z_{1}\right)+\lambda_{9}\left(z_{0}, z_{1}\right)}{1-\left(\lambda_{2}\left(z_{0}, z_{1}\right)+\lambda_{5}\left(z_{0}, z_{1}\right)+2 \lambda_{6}\left(z_{0}, z_{1}\right)+\lambda_{8}\left(z_{0}, z_{1}\right)+\lambda_{9}\left(z_{0}, z_{1}\right)\right)} .
$$

Consequently,

$$
\left|d\left(z_{2 k+1}, z_{2 k}\right)\right| \leq h\left|d\left(z_{2 k}, z_{2 k-1}\right)\right|,
$$


where

$$
h=\frac{\lambda_{1}\left(z_{0}, z_{1}\right)+\sum_{i=4}^{9} \lambda_{i}\left(z_{0}, z_{1}\right)}{1-\left(\sum_{i=2}^{5} \lambda_{i}\left(z_{0}, z_{1}\right)+2\left[\lambda_{6}\left(z_{0}, z_{1}\right)+\lambda_{8}\left(z_{0}, z_{1}\right)\right]+\lambda_{7}\left(z_{0}, z_{1}\right)+\lambda_{9}\left(z_{0}, z_{1}\right)\right)} .
$$

We rewrite lines $15-21$ on page 103 and lines 1,2 on page 104 as follows

$$
\left|d\left(z_{2 k-1}, z_{2 k}\right)\right| \leq h_{1}\left|d\left(z_{2 k-2}, z_{2 k-1}\right)\right|,
$$

where

$$
h_{1}=\frac{\lambda_{1}\left(z_{0}, z_{1}\right)+\lambda_{4}\left(z_{0}, z_{1}\right)+\lambda_{7}\left(z_{0}, z_{1}\right)+\lambda_{8}\left(z_{0}, z_{1}\right)}{1-\left(\lambda_{2}\left(z_{0}, z_{1}\right)+\lambda_{4}\left(z_{0}, z_{1}\right)+\lambda_{6}\left(z_{0}, z_{1}\right)+\lambda_{7}\left(z_{0}, z_{1}\right)+2 \lambda_{8}\left(z_{0}, z_{1}\right)\right)},
$$

which implies that

$$
\left|d\left(z_{2 k-1}, z_{2 k}\right)\right| \leq h\left|d\left(z_{2 k-2}, z_{2 k-1}\right)\right| .
$$

Now, from equations (4) and (5), we have

$$
\left|d\left(z_{2 k+1}, z_{2 k}\right)\right| \leq h\left|d\left(z_{2 k}, z_{2 k-1}\right)\right| \leq h^{2}\left|d\left(z_{2 k-1}, z_{2 k-2}\right)\right| .
$$

Replace $y$ by $w$ in line 15 on page 104 and replace $x$ by $z, y$ by $w$ in line 1 on page 106.

Now, we present the correct version of Theorem 3.7 ([1]).

TheOREM 2. Let $(X, d)$ be a complete complex valued metric space and $\lambda_{i}: X \times X \rightarrow[0,1), i=1,2, \ldots, 9$. Assume that $K, L: X \rightarrow X$ are two self-mappings such that for all $z, w \in X$ the following conditions are satisfied:

(i) $\lambda_{i}(L K z, w) \leq \lambda_{i}(z, w)$ and $\lambda_{i}(z, K L w) \leq \lambda_{i}(z, w), i=1, \ldots, 9$;

(ii) $d(K z, L w) \precsim \lambda_{1}(z, w) d(z, w)+\lambda_{2}(z, w) \frac{d(z, K z) d(w, L w)}{1+d(z, w)}$

$$
\begin{aligned}
& +\lambda_{3}(z, w) \frac{d(w, K z) d(z, L w)}{1+d(z, w)}+\lambda_{4}(z, w) \frac{d(z, K z) d(z, L w)}{1+d(z, w)} \\
& +\lambda_{5}(z, w) \frac{d(w, K z) d(w, L w)}{1+d(z, w)} \\
& +\lambda_{6}(z, w) \frac{d(w, L w)[d(z, K z)+d(w, K z)]}{1+d(z, w)+d(K z, L w)}
\end{aligned}
$$




$$
\begin{aligned}
& +\lambda_{7}(z, w) \frac{d(z, L w)[d(z, K z)+d(w, K z)]}{1+d(z, w)+d(K z, L w)} \\
& +\lambda_{8}(z, w) \frac{d(z, K z)[d(z, L w)+d(w, L w)]}{1+d(z, w)+d(K z, L w)} \\
& +\lambda_{9}(z, w) \frac{d(w, K z)[d(z, L w)+d(w, L w)]}{1+d(z, w)+d(K z, L w)}
\end{aligned}
$$

where

$$
\begin{aligned}
\sum_{i=1}^{3} \lambda_{i}(z, w)+2\left[\lambda_{4}(z, w)+\lambda_{5}(z, w)+\lambda_{7}(z, w)\right. & \left.+\lambda_{9}(z, w)\right] \\
+ & 3\left[\lambda_{6}(z, w)+\lambda_{8}(z, w)\right]<1
\end{aligned}
$$

Then $K$ and $L$ have a unique common fixed point in $X$.

Proof. Let $z_{0} \in X$ and let us construct a sequence $\left\{z_{n}\right\}$ by the rule

$$
K z_{2 n}=z_{2 n+1} \quad \text { and } \quad L z_{2 n+1}=z_{2 n+2}, \quad n=0,1,2, \ldots
$$

First we show that $\left\{z_{n}\right\}$ is a Cauchy sequence in $X$. For this, consider

$$
d\left(z_{2 k+1}, z_{2 k}\right)=d\left(K L z_{2 k-1}, L z_{2 k-1}\right) .
$$

By using condition (ii) of Theorem 2 with $z=L z_{2 k-1}, w=z_{2 k-1}$ and equations (6), we get

$$
\begin{aligned}
d\left(z_{2 k+1}, z_{2 k}\right) \precsim & \lambda_{1}\left(z_{2 k}, z_{2 k-1}\right) d\left(z_{2 k}, z_{2 k-1}\right) \\
& +\lambda_{2}\left(z_{2 k}, z_{2 k-1}\right) \frac{d\left(z_{2 k}, z_{2 k+1}\right) d\left(z_{2 k-1}, z_{2 k}\right)}{1+d\left(z_{2 k}, z_{2 k-1}\right)} \\
& +\lambda_{3}\left(z_{2 k}, z_{2 k-1}\right) \frac{d\left(z_{2 k-1}, z_{2 k+1}\right) d\left(z_{2 k}, z_{2 k}\right)}{1+d\left(z_{2 k}, z_{2 k-1}\right)} \\
& +\lambda_{4}\left(z_{2 k}, z_{2 k-1}\right) \frac{d\left(z_{2 k}, z_{2 k+1}\right) d\left(z_{2 k}, z_{2 k}\right)}{1+d\left(z_{2 k}, z_{2 k-1}\right)} \\
& +\lambda_{5}\left(z_{2 k}, z_{2 k-1}\right) \frac{d\left(z_{2 k-1}, z_{2 k+1}\right) d\left(z_{2 k-1}, z_{2 k}\right)}{1+d\left(z_{2 k}, z_{2 k-1}\right)} \\
& +\lambda_{6}\left(z_{2 k}, z_{2 k-1}\right) \frac{d\left(z_{2 k-1}, z_{2 k}\right)\left[d\left(z_{2 k}, z_{2 k+1}\right)+d\left(z_{2 k-1}, z_{2 k+1}\right)\right]}{1+d\left(z_{2 k}, z_{2 k-1}\right)+d\left(z_{2 k+1}, z_{2 k}\right)}
\end{aligned}
$$




$$
\begin{aligned}
& +\lambda_{7}\left(z_{2 k}, z_{2 k-1}\right) \frac{d\left(z_{2 k}, z_{2 k}\right)\left[d\left(z_{2 k}, z_{2 k+1}\right)+d\left(z_{2 k-1}, z_{2 k+1}\right)\right]}{1+d\left(z_{2 k}, z_{2 k-1}\right)+d\left(z_{2 k+1}, z_{2 k}\right)} \\
& +\lambda_{8}\left(z_{2 k}, z_{2 k-1}\right) \frac{d\left(z_{2 k}, z_{2 k+1}\right)\left[d\left(z_{2 k}, z_{2 k}\right)+d\left(z_{2 k-1}, z_{2 k}\right)\right]}{1+d\left(z_{2 k}, z_{2 k-1}\right)+d\left(z_{2 k+1}, z_{2 k}\right)} \\
& +\lambda_{9}\left(z_{2 k}, z_{2 k-1}\right) \frac{d\left(z_{2 k-1}, z_{2 k+1}\right)\left[d\left(z_{2 k}, z_{2 k}\right)+d\left(z_{2 k-1}, z_{2 k}\right)\right]}{1+d\left(z_{2 k}, z_{2 k-1}\right)+d\left(z_{2 k+1}, z_{2 k}\right)} .
\end{aligned}
$$

From Proposition 2.1 in [2] and triangle inequality, we can write

$$
\begin{aligned}
\left|d\left(z_{2 k+1}, z_{2 k}\right)\right| \leq & \lambda_{1}\left(z_{0}, z_{1}\right)\left|d\left(z_{2 k}, z_{2 k-1}\right)\right|+\lambda_{2}\left(z_{0}, z_{1}\right)\left|d\left(z_{2 k}, z_{2 k+1}\right)\right| \\
& +\lambda_{5}\left(z_{0}, z_{1}\right)\left|d\left(z_{2 k-1}, z_{2 k}\right)\right|+\lambda_{5}\left(z_{0}, z_{1}\right)\left|d\left(z_{2 k}, z_{2 k+1}\right)\right| \\
& +\lambda_{6}\left(z_{0}, z_{1}\right)\left|d\left(z_{2 k}, z_{2 k+1}\right)\right|+\lambda_{6}\left(z_{0}, z_{1}\right)\left|d\left(z_{2 k-1}, z_{2 k}\right)\right| \\
& +\lambda_{6}\left(z_{0}, z_{1}\right)\left|d\left(z_{2 k}, z_{2 k+1}\right)\right|+\lambda_{8}\left(z_{0}, z_{1}\right)\left|d\left(z_{2 k}, z_{2 k+1}\right)\right| \\
& +\lambda_{9}\left(z_{0}, z_{1}\right)\left|d\left(z_{2 k-1}, z_{2 k}\right)\right|+\lambda_{9}\left(z_{0}, z_{1}\right)\left|d\left(z_{2 k}, z_{2 k+1}\right)\right| .
\end{aligned}
$$

Finally, one can get

$$
\left|d\left(z_{2 k+1}, z_{2 k}\right)\right| \leq h_{0}\left|d\left(z_{2 k}, z_{2 k-1}\right)\right|
$$

where

$$
h_{0}=\frac{\lambda_{1}\left(z_{0}, z_{1}\right)+\lambda_{5}\left(z_{0}, z_{1}\right)+\lambda_{6}\left(z_{0}, z_{1}\right)+\lambda_{9}\left(z_{0}, z_{1}\right)}{1-\left(\lambda_{2}\left(z_{0}, z_{1}\right)+\lambda_{5}\left(z_{0}, z_{1}\right)+2 \lambda_{6}\left(z_{0}, z_{1}\right)+\lambda_{8}\left(z_{0}, z_{1}\right)+\lambda_{9}\left(z_{0}, z_{1}\right)\right)} .
$$

Consequently,

$$
\left|d\left(z_{2 k+1}, z_{2 k}\right)\right| \leq h\left|d\left(z_{2 k}, z_{2 k-1}\right)\right|
$$

where

$$
h=\frac{\lambda_{1}\left(z_{0}, z_{1}\right)+\sum_{i=4}^{9} \lambda_{i}\left(z_{0}, z_{1}\right)}{1-\left(\sum_{i=2}^{5} \lambda_{i}\left(z_{0}, z_{1}\right)+2\left[\lambda_{6}\left(z_{0}, z_{1}\right)+\lambda_{8}\left(z_{0}, z_{1}\right)\right]+\lambda_{7}\left(z_{0}, z_{1}\right)+\lambda_{9}\left(z_{0}, z_{1}\right)\right)} .
$$

On the other hand, consider

$$
d\left(z_{2 k-1}, z_{2 k}\right)=d\left(K z_{2 k-2}, L K z_{2 k-2}\right) .
$$


By applying condition (ii) of Theorem 2 with $z=z_{2 k-2}$ and $w=K z_{2 k-2}$, equation (8) and using equations (6) and Proposition 2.1 in [2], one can get

$$
\begin{aligned}
\left|d\left(z_{2 k-1}, z_{2 k}\right)\right| \leq & \lambda_{1}\left(z_{0}, z_{1}\right)\left|d\left(z_{2 k-2}, z_{2 k-1}\right)\right| \\
& +\lambda_{2}\left(z_{0}, z_{1}\right)\left|\frac{d\left(z_{2 k-2}, z_{2 k-1}\right) d\left(z_{2 k-1}, z_{2 k}\right)}{1+d\left(z_{2 k-2}, z_{2 k-1}\right)}\right| \\
& +\lambda_{4}\left(z_{0}, z_{1}\right)\left|\frac{d\left(z_{2 k-2}, z_{2 k-1}\right) d\left(z_{2 k-2}, z_{2 k}\right)}{1+d\left(z_{2 k-2}, z_{2 k-1}\right)}\right| \\
& +\lambda_{6}\left(z_{0}, z_{1}\right)\left|\frac{d\left(z_{2 k-1}, z_{2 k}\right) d\left(z_{2 k-2}, z_{2 k-1}\right)}{1+d\left(z_{2 k-2}, z_{2 k-1}\right)+d\left(z_{2 k-1}, z_{2 k}\right)}\right| \\
& +\lambda_{7}\left(z_{0}, z_{1}\right)\left|\frac{d\left(z_{2 k-2}, z_{2 k}\right) d\left(z_{2 k-2}, z_{2 k-1}\right)}{1+d\left(z_{2 k-2}, z_{2 k-1}\right)+d\left(z_{2 k-1}, z_{2 k}\right)}\right| \\
& +\lambda_{8}\left(z_{0}, z_{1}\right)\left|\frac{d\left(z_{2 k-2}, z_{2 k-1}\right)\left[d\left(z_{2 k-2}, z_{2 k}\right)+d\left(z_{2 k-1}, z_{2 k}\right)\right]}{1+d\left(z_{2 k-2}, z_{2 k-1}\right)+d\left(z_{2 k-1}, z_{2 k}\right)}\right| \\
\leq & \lambda_{1}\left(z_{0}, z_{1}\right)\left|d\left(z_{2 k-2}, z_{2 k-1}\right)\right|+\lambda_{2}\left(z_{0}, z_{1}\right)\left|d\left(z_{2 k-1}, z_{2 k}\right)\right| \\
& +\lambda_{4}\left(z_{0}, z_{1}\right)\left|d\left(z_{2 k-2}, z_{2 k}\right)\right|+\lambda_{6}\left(z_{0}, z_{1}\right)\left|d\left(z_{2 k-1}, z_{2 k}\right)\right| \\
& +\lambda_{7}\left(z_{0}, z_{1}\right)\left|d\left(z_{2 k-2}, z_{2 k}\right)\right|+\lambda_{8}\left(z_{0}, z_{1}\right)\left|d\left(z_{2 k-2}, z_{2 k}\right)\right| \\
& +\lambda_{8}\left(z_{0}, z_{1}\right)\left|d\left(z_{2 k-1}, z_{2 k}\right)\right| \\
\leq & \lambda_{1}\left(z_{0}, z_{1}\right)\left|d\left(z_{2 k-2}, z_{2 k-1}\right)\right|+\lambda_{2}\left(z_{0}, z_{1}\right)\left|d\left(z_{2 k-1}, z_{2 k}\right)\right| \\
& +\lambda_{4}\left(z_{0}, z_{1}\right)\left|d\left(z_{2 k-2}, z_{2 k-1}\right)\right|+\lambda_{4}\left(z_{0}, z_{1}\right)\left|d\left(z_{2 k-1}, z_{2 k}\right)\right| \\
& +\lambda_{6}\left(z_{0}, z_{1}\right)\left|d\left(z_{2 k-1}, z_{2 k}\right)\right|+\lambda_{7}\left(z_{0}, z_{1}\right)\left|d\left(z_{2 k-2}, z_{2 k-1}\right)\right| \\
& +\lambda_{7}\left(z_{0}, z_{1}\right)\left|d\left(z_{2 k-1}, z_{2 k}\right)\right|+\lambda_{8}\left(z_{0}, z_{1}\right)\left|d\left(z_{2 k-2}, z_{2 k-1}\right)\right| \\
& +\lambda_{8}\left(z_{0}, z_{1}\right)\left|d\left(z_{2 k-1}, z_{2 k}\right)\right|+\lambda_{8}\left(z_{0}, z_{1}\right)\left|d\left(z_{2 k-1}, z_{2 k}\right)\right| \\
&
\end{aligned}
$$

This implies that

$$
\left|d\left(z_{2 k-1}, z_{2 k}\right)\right| \leq h_{1}\left|d\left(z_{2 k-2}, z_{2 k-1}\right)\right| \leq h\left|d\left(z_{2 k-2}, z_{2 k-1}\right)\right|,
$$

where

$$
h_{1}=\frac{\lambda_{1}\left(z_{0}, z_{1}\right)+\lambda_{4}\left(z_{0}, z_{1}\right)+\lambda_{7}\left(z_{0}, z_{1}\right)+\lambda_{8}\left(z_{0}, z_{1}\right)}{1-\left(\lambda_{2}\left(z_{0}, z_{1}\right)+\lambda_{4}\left(z_{0}, z_{1}\right)+\lambda_{6}\left(z_{0}, z_{1}\right)+\lambda_{7}\left(z_{0}, z_{1}\right)+2 \lambda_{8}\left(z_{0}, z_{1}\right)\right)} .
$$


Now, from equations (7) and (9), we have

$$
\left|d\left(z_{2 k+1}, z_{2 k}\right)\right| \leq h\left|d\left(z_{2 k}, z_{2 k-1}\right)\right| \leq h^{2}\left|d\left(z_{2 k-1}, z_{2 k-2}\right)\right|
$$

Consequently, we can write

$$
\left|d\left(z_{n+1}, z_{n}\right)\right| \leq h\left|d\left(z_{n}, z_{n-1}\right)\right| \leq h^{2}\left|d\left(z_{n-1}, z_{n-2}\right)\right| \leq \ldots \leq h^{n}\left|d\left(z_{1}, z_{0}\right)\right|
$$

Now, for $m>n$,

$$
d\left(z_{n}, z_{m}\right) \precsim d\left(z_{n}, z_{n+1}\right)+d\left(z_{n+1}, z_{n+2}\right)+\ldots+d\left(z_{m-1}, z_{m}\right)
$$

Thus $\left|d\left(z_{n}, z_{m}\right)\right| \leq \frac{h^{n}}{1-h}\left|d\left(z_{1}, z_{0}\right)\right|$, which implies that $\lim _{n \rightarrow \infty}\left|d\left(z_{n}, z_{m}\right)\right|=0$. Hence, $\left\{z_{n}\right\}$ is a Cauchy sequence. But $X$ is complete, so there exists $t \in X$ such that $z_{n} \rightarrow t$ as $n \rightarrow \infty$.

Next, we show that $t$ is a fixed point of $K$. For this, consider

$$
d(t, K t) \precsim d\left(t, L z_{2 n+1}\right)+d\left(L z_{2 n+1}, K t\right) .
$$

Using condition (ii) of Theorem 2 with $z=t$ and $w=z_{2 n+1}$, equation (6) and Proposition 2.1 in [2], we get

$$
\begin{aligned}
d(t, K t) \precsim & d\left(t, z_{2 n+2}\right)+\lambda_{1}\left(t, z_{1}\right) d\left(t, z_{2 n+1}\right)+\lambda_{2}\left(t, z_{1}\right) \frac{d(t, K t) d\left(z_{2 n+1}, z_{2 n+2}\right)}{1+d\left(t, z_{2 n+1}\right)} \\
& +\lambda_{3}\left(t, z_{1}\right) \frac{d\left(z_{2 n+1}, K t\right) d\left(t, z_{2 n+2}\right)}{1+d\left(t, z_{2 n+1}\right)}+\lambda_{4}\left(t, z_{1}\right) \frac{d(t, K t) d\left(t, z_{2 n+2}\right)}{1+d\left(t, z_{2 n+1}\right)} \\
& +\lambda_{5}\left(t, z_{1}\right) \frac{d\left(z_{2 n+1}, K t\right) d\left(z_{2 n+1}, z_{2 n+2}\right)}{1+d\left(t, z_{2 n+1}\right)} \\
& +\lambda_{6}\left(t, z_{1}\right) \frac{d\left(z_{2 n+1}, z_{2 n+2}\right)\left[d(t, K t)+d\left(z_{2 n+1}, K t\right)\right]}{1+d\left(t, z_{2 n+1}\right)+d\left(K t, z_{2 n+2}\right)} \\
& +\lambda_{7}\left(t, z_{1}\right) \frac{d\left(t, z_{2 n+2}\right)\left[d(t, K t)+d\left(z_{2 n+1}, K t\right)\right]}{1+d\left(t, z_{2 n+1}\right)+d\left(K t, z_{2 n+2}\right)} \\
& +\lambda_{8}\left(t, z_{1}\right) \frac{d(t, K t)\left[d\left(t, z_{2 n+2}\right)+d\left(z_{2 n+1}, z_{2 n+2}\right)\right]}{1+d\left(t, z_{2 n+1}\right)+d\left(K t, z_{2 n+2}\right)} \\
& +\lambda_{9}\left(t, z_{1}\right) \frac{d\left(z_{2 n+1}, K t\right)\left[d\left(t, z_{2 n+2}\right)+d\left(z_{2 n+1}, z_{2 n+2}\right)\right]}{1+d\left(t, z_{2 n+1}\right)+d\left(K t, z_{2 n+2}\right)} .
\end{aligned}
$$

Taking limit as $n \rightarrow \infty$, we get $d(K t, t) \precsim 0$, whence $d(K t, t)=0$. Thus $K t=t$ and hence $t$ is a fixed point of $K$. 
Similarly, using condition (ii) of Theorem 2 with $z=z_{2 n}$ and $w=t$ one can show that $t$ is a fixed point of $L$. Therefore $K t=L t=t$, that is $t$ is a common fixed point of $K$ and $L$.

For uniqueness, suppose that $t^{*} \neq t$ is another fixed point of $K$ and $L$. Then putting $z=t$ and $w=t^{*}$ in condition (ii) of Theorem 2, we have

$$
\begin{aligned}
d\left(t, t^{*}\right)= & d\left(K t, L t^{*}\right) \\
\precsim & \lambda_{1}\left(t, t^{*}\right) d\left(t, t^{*}\right)+\lambda_{2}\left(t, t^{*}\right) \frac{d(t, K t) d\left(t^{*}, L t^{*}\right)}{1+d\left(t, t^{*}\right)} \\
& +\lambda_{3}\left(t, t^{*}\right) \frac{d\left(t^{*}, K t\right) d\left(t, L t^{*}\right)}{1+d\left(t, t^{*}\right)}+\lambda_{4}\left(t, t^{*}\right) \frac{d(t, K t) d\left(t, L t^{*}\right)}{1+d\left(t, t^{*}\right)} \\
& +\lambda_{5}\left(t, t^{*}\right) \frac{d\left(t^{*}, K t\right) d\left(t^{*}, L t^{*}\right)}{1+d\left(t, t^{*}\right)} \\
& +\lambda_{6}\left(t, t^{*}\right) \frac{d\left(t^{*}, L t^{*}\right)\left[d(t, K t)+d\left(t^{*}, K t\right)\right]}{1+d\left(t, t^{*}\right)+d\left(K t, L t^{*}\right)} \\
& +\lambda_{7}\left(t, t^{*}\right) \frac{d\left(t, L t^{*}\right)\left[d(t, K t)+d\left(t^{*}, K t\right)\right]}{1+d\left(t, t^{*}\right)+d\left(K t, L t^{*}\right)} \\
& +\lambda_{8}\left(t, t^{*}\right) \frac{d(t, K t)\left[d\left(t, L t^{*}\right)+d\left(t^{*}, L t^{*}\right)\right]}{1+d\left(t, t^{*}\right)+d\left(K t, L t^{*}\right)} \\
& +\lambda_{9}\left(t, t^{*}\right) \frac{d\left(t^{*}, K t\right)\left[d\left(t, L t^{*}\right)+d\left(t^{*}, L t^{*}\right)\right]}{1+d\left(t, t^{*}\right)+d\left(K t, L t^{*}\right)}
\end{aligned}
$$

which implies that

$$
\begin{aligned}
\left|d\left(t, t^{*}\right)\right| \leq & \lambda_{1}\left(t, t^{*}\right)\left|d\left(t, t^{*}\right)\right|+\lambda_{3}\left(t, t^{*}\right)\left|\frac{d\left(t^{*}, t\right) d\left(t, t^{*}\right)}{1+d\left(t, t^{*}\right)}\right| \\
& +\lambda_{7}\left(t, t^{*}\right)\left|\frac{d\left(t, t^{*}\right) d\left(t^{*}, t\right)}{1+d\left(t, t^{*}\right)+d\left(t, t^{*}\right)}\right|+\lambda_{9}\left(t, t^{*}\right)\left|\frac{d\left(t^{*}, t\right) d\left(t, t^{*}\right)}{1+d\left(t, t^{*}\right)+d\left(t, t^{*}\right)}\right| \\
\leq & {\left[\lambda_{1}\left(t, t^{*}\right)+\lambda_{3}\left(t, t^{*}\right)+\lambda_{7}\left(t, t^{*}\right)+\lambda_{9}\left(t, t^{*}\right)\right]\left|d\left(t^{*}, t\right)\right| . }
\end{aligned}
$$

This is a contradiction, thus $t^{*}=t$. Hence $t$ is a unique common fixed point of $K$ and $L$.

In the proof of Lemma 3.21 replace $x_{n}$ by $z_{n}$ and replace $L K$ by $K L$ in line 23 on page 108.

Now we recall Lemma 3.21 ([1]) and formulate its corrected proof. 
Lemma 3. Let $(X, d)$ be a complete complex valued metric space and $K, L: X \rightarrow X$. Let $z_{0} \in X$ and define the sequence $\left\{z_{n}\right\}$ by

$$
K z_{2 n}=z_{2 n+1} \quad \text { and } \quad L z_{2 n+1}=z_{2 n+2}, \quad n=0,1,2, \ldots
$$

Assume that there exists a mapping $\lambda: X \rightarrow[0,1)$ satisfying

$$
\lambda(L K z) \leq \lambda(z) \quad \text { and } \quad \lambda(K L z) \leq \lambda(z) \quad \text { for all } z \in X .
$$

Then $\lambda\left(z_{2 n}\right) \leq \lambda\left(z_{0}\right)$ and $\lambda\left(z_{2 n+1}\right) \leq \lambda\left(z_{1}\right)$ for all $z \in X$ and $n=0,1,2, \ldots$

Proof. Let $n \in\{0,1,2,3, \ldots\}$. We have

$$
\begin{aligned}
\lambda\left(z_{2 n}\right) & =\lambda\left(L K z_{2 n-2}\right) \leq \lambda\left(z_{2 n-2}\right)=\lambda\left(L K z_{2 n-4}\right) \\
& \leq \lambda\left(z_{2 n-4}\right) \leq \ldots \leq \lambda\left(z_{0}\right) .
\end{aligned}
$$

Similarly,

$$
\begin{aligned}
\lambda\left(z_{2 n+1}\right) & =\lambda\left(K L z_{2 n-1}\right) \leq \lambda\left(z_{2 n-1}\right)=\lambda\left(K L z_{2 n-3}\right) \\
& \leq \lambda\left(z_{2 n-3}\right) \leq \ldots \leq \lambda\left(z_{1}\right) .
\end{aligned}
$$

In Theorem 3.22 the denominator of the rational expressions in lines 4-7 on page 109 should be corrected as

$$
1+d(z, w)+d(K z, L w) \text { instead of } 1+d(z, w) .
$$

The new version of Theorem 3.22 ([1]) reads as follows.

TheOREM 4. Let $(X, d)$ be a complete complex valued metric space and $\lambda_{i}: X \rightarrow[0,1), i=1,2, \ldots, 9$. Assume that $K, L: X \rightarrow X$ are two selfmappings such that for all $z, w \in X$ the following conditions are satisfied:

(i) $\lambda_{i}(L K z) \leq \lambda_{i}(z)$ and $\lambda_{i}(K L z) \leq \lambda_{i}(z), i=1, \ldots, 9$;

(ii) $d(K z, L w) \precsim \lambda_{1}(z) d(z, w)$

$$
\begin{aligned}
& +\lambda_{2}(z) \frac{d(z, K z) d(w, L w)}{1+d(z, w)}+\lambda_{3}(z) \frac{d(w, K z) d(z, L w)}{1+d(z, w)} \\
& +\lambda_{4}(z) \frac{d(z, K z) d(z, L w)}{1+d(z, w)}+\lambda_{5}(z) \frac{d(w, K z) d(w, L w)}{1+d(z, w)} \\
& +\lambda_{6}(z) \frac{d(w, L w)[d(z, K z)+d(w, K z)]}{1+d(z, w)+d(K z, L w)}
\end{aligned}
$$




$$
\begin{aligned}
& +\lambda_{7}(z) \frac{d(z, L w)[d(z, K z)+d(w, K z)]}{1+d(z, w)+d(K z, L w)} \\
& +\lambda_{8}(z) \frac{d(z, K z)[d(z, L w)+d(w, L w)]}{1+d(z, w)+d(K z, L w)} \\
& +\lambda_{9}(z) \frac{d(w, K z)[d(z, L w)+d(w, L w)]}{1+d(z, w)+d(K z, L w)}
\end{aligned}
$$

where

$$
\sum_{i=1}^{3} \lambda_{i}(z)+2\left[\lambda_{4}(z)+\lambda_{5}(z)+\lambda_{7}(z)+\lambda_{9}(z)\right]+3\left[\lambda_{6}(z)+\lambda_{8}(z)\right]<1 .
$$

Then $K$ and $L$ have a unique common fixed point in $X$.

Acknowledgements. The authors wish to thank the editor and anonymous referees for their valuable comments and suggestions, which helped to improve this manuscript.

\section{References}

[1] Kumam P., Sarwar M., Zada M.B., Fixed point results satisfying rational type contractive conditions in complex valued metric spaces, Ann. Math. Sil. 30 (2016), 89-110.

[2] Sitthikul K., Saejung S., Some fixed point theorems in complex valued metric space, Fixed Point Theory Appl. 2012, 2012:189, 11 pp.

\footnotetext{
Department of Mathematics

UNIVERSITY OF MALAKAND

Chakdara Dir(L)

PAKISTAN

e-mail: sarwarswati@gmail.com

e-mail: mbz.math@gmail.com
} 